\title{
Studying Magnetization Distribution in Magnetic Thin Films under Transversal Application of Magnetic Fields
}

\author{
Aleksey Anatolievich Zakharenko \\ International Institute of Zakharenko Waves, Krasnoyarsk, Russia \\ E-mail:aazaaz@inbox.ru \\ Received January 25, 2010; revised February 26, 2010; accepted March 20, 2010
}

\begin{abstract}
The problem of magnetization change across the direction of magnetic field for a magnetic layer with non-symmetric boundary conditions was treated. The exact solution of the problem for the magnetization components $m_{x}$ and $m_{y}$ was written in the form of complex combination of Jacobian elliptic functions and elliptic integrals. This allows one to demonstrate both the static mode and all dynamic modes for the magnetization distribution across the layer thickness. The static mode and several dynamic modes, as well as the first and second derivatives of the magnetization components, were calculated. Also, average values of the magnetization components $\left\langle m_{x}\right\rangle$ and $\left\langle m_{y}\right\rangle$ for the static mode and three dynamic modes were calculated in dependence on the magnetic field. The obtained results can represent an interest in the large amount of applications of magnetic devices such as recording media, memory chips, and computer disks. The results are also useful for checking different numerical methods recently applied to study the problem, because it is thought that any numerical method cannot demonstrate solutions for the dynamic modes.
\end{abstract}

Keywords: Landau-Lifshits Equations, Thin Films, Magnetization, Static and Dynamic Modes.

\section{Introduction}

The Landau-Lifshits equations first derived by Landau and Lifshits on a phenomenological ground in $[1,2]$ are fundamental equations in the theory of ferromagnetism. The study of the equations can demonstrate the magnetization distribution inside a ferromagnetic material and is a very challenging problem in physics and mathematics. Indeed, the study of them can be useful for a set of applications of magnetic devices $[3,4]$ such as recording media and computer sensors, disks, and memory chips. The numerical and theoretical studies of the equations can be found in many works carried out in the last several decades (for example see [3-10]). However, it is thought that any numerical treatment can not demonstrate existence of a set of additional solutions. These solutions can improve understanding of magnetization distributions in a ferromagnetic layer when different regimes of application of magnetic fields can be realized for a domain in the layer.

It is assumed that in a ferromagnetic layer on an anti-ferromagnetic substrate, the vector of magnetic momentum $\mathbf{M}$ can be clamped at the boundary between the layer and the substrate. In this two-layer system, some magnetic structures [11,12] can appear when an external magnetic field is applied in the layer plane.
These magnetic structures are characterized by inhomogeneous turn of the M across the layer thickness. In 1980, Zakharov and Khlebopros [13] reported that solutions written in [12] for the magnetization distribution in a magnetically-soft layer on a magnetically-hard substrate under axial application of a magnetic field can be written in a novel way. Also, some solutions of the problem were originally found by Aharoni [11] in 1959, using the Jacobian elliptic functions $[14,15]$. Also, the excellent and classical book [16] by Collatz provides solutions of eigenvalue problems with different boundary conditions.

In 1995, the further theoretical investigations by Zakharov [17] considered the magnetic reversal of a magnetic system in a layer across anisotropy. Studying one of the magnetization components of the first dynamic mode, it was shown that magnetization in the layer after the dynamic threshold can turn from the equilibrium position and becomes opposite to the field over the entire thickness of the layer. It was also assumed in [17] that this occurs in a similar manner as a rod loaded by a transverse force is bent oppositely to the force direction as soon as the first dynamic threshold is achieved. Note that an infinite number of all higher-order modes followed the single static mode can be called the dynamic modes. In 1949, this definition of dynamic loss of stability was first given by Lavrent'ev and Ishlinskii [18] 
when they studied a shock loading applied to rods. Indeed, the problem of magnetization change along the direction of magnetic anisotropy of a magnetic layer with non-symmetric boundary conditions is similar to the Euler problem of stability of an elastic rod. Note that exact solutions for the problem of transversal loading of the rods were recently given in the work [19]. It is thought that magnetic systems are more convenient for studying the dynamic buckling because of many experiments on these systems which can be performed.

It is also thought that these magnetic layered structures can represent an interest in the development of idea of creation of the metallic transistor [20-22] because an applied magnetic field can easily create an inhomogeneous distribution of the magnetization. Indeed, it is possible to study some effects resulting from the problem. Also, it is thought that the theoretical studies can be important for grasping some processes when liquid crystals and seignette-electrics are switched by super-strong fields. Some interesting experimental data can be found in the review paper [23] for the novel evaluation of the problem.

This theoretical study of the magnetization distribution provides exact solutions leading to existence possibility of infinite number of dynamic modes in addition to the single static mode. In the studied case, the magnetic field is transversely applied. The following section describes the theory. The third section investigates the magnetization components concerning extreme and inflexion points. In addition, the fourth section provides the magnetization distribution in the case when the magnetic anisotropy is accounted.

\section{Theory}

The magnetic layered system is shown in Figure 1 when a ferromagnetic layer with the thickness $d$ is situated on an anti-ferromagnetic substrate. The $z$-axis is directed parallel to the normal to both the layer surface and the interface between the layers; $z=0$ at the interface. The $x$-axis and $y$-axis lie in the plane of the interface. It is

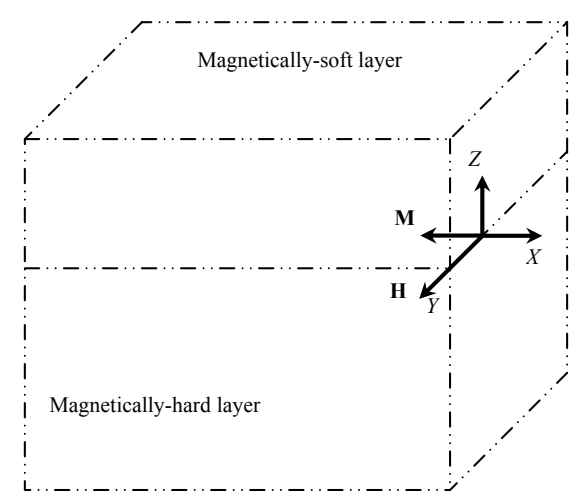

Figure 1. The configuration of a magnetically-soft layer on a magnetically-hard layer. possible to treat domains with equal width and with negligibly thin walls such that the walls' energy can be omitted. The applied magnetic field $\mathbf{H}$ is directed along the $y$-axis, and the initial direction of the magnetization vector $\mathbf{M}$ is towards the $x$-axis negative values as shown in the figure. The applied $\mathbf{H}$ can turn the magnetization vector $\mathbf{M}$.

The Landau-Lifshitz Equation (1) can be written in the following form [13]:

$$
\dot{\mathbf{M}}_{j}=g \mathbf{M}_{j} \times \mathbf{H}_{j}^{(e)}
$$

where $\times$ is the vector cross product, $g$ is the exchange coupling constant (gyromagnetic ratio); $j=1,2$. In Equation (1), the term on the left represents the first derivative of $\mathbf{M}_{j}$ with respect to time. The boundary conditions for Equation (1) are chosen as follows:

$$
M_{i x}=-M, M_{i y}=M_{i z}=0 \text { at } z=0 \text { and } \partial \mathbf{M}_{i} / \partial n=0 \text { at } z=d
$$

when the $n$ is directed along the surface normal. In Equation (1), the effective magnetic fields $\mathbf{H}_{i}^{(e)}$ can be written in the following form for this case:

$$
\mathbf{H}_{i}^{(e)}=\alpha \nabla^{2} \mathbf{M}_{i}+\mathbf{H}-2 \frac{\partial E_{d m}}{\partial \mathbf{M}_{i}}
$$

where $\alpha$ is the constant of exchange for a ferromagnetics, $\mathbf{H}$ represents external constant and altering magnetic fields. The energy $E_{d m}$ related to demagnetization fields existing at the domain boundaries can be written in the form of [13]:

$$
\begin{gathered}
E_{d m}=\frac{1}{8}\left[4 \pi\left(M_{1 z}+M_{2 z}\right)^{2}+\eta_{x}\left(M_{1 x}-M_{2 x}\right)^{2}+\right. \\
\left.-\eta_{y}\left(M_{1 y}-M_{2 y}\right)^{2}+\eta_{z}\left(M_{1 z}-M_{2 z}\right)^{2}\right]
\end{gathered}
$$

where the demagnetization factors $\eta_{j}$ are as follows: $\eta_{x}=$ $0, \eta_{y}=4 \pi d /(d+D), \eta_{z}=4 \pi D /(d+D)$ [13] with $d$ and $D$ representing the layer thickness and domain width, respectively. It is possible to use normalized field $\mathbf{h}=\mathbf{H} / M$ and normalized magnetization $\mathbf{m}^{*}{ }_{i}=\mathbf{M}_{i} / M$. The $\mathbf{m}^{*}{ }_{i}$ depend on the coordinate $z$ and time $t$, and can be written as corresponding static and dynamic terms: $\mathbf{m}^{*}{ }_{i}(z, t)=\mathbf{m}_{i}(z)$ $+\boldsymbol{\mu}_{i}(z, t)$. The dynamic $\boldsymbol{\mu}_{i}(z, t)$ were treated in [13] and do not represent a studying subject of this work. Note that in the treated case, the static magnetization components of $\mathbf{m}_{i}(z)$ satisfy the following relationships:

$$
\begin{gathered}
m_{1 x}=m_{2 x}=m_{x}, m_{1 y}=-m_{2 y}=m_{y}, m_{1 z}=m_{2 z}=0, \\
m_{x}^{2}+m_{y}^{2}=1
\end{gathered}
$$

After several complicated mathematical transformations described in [13] and accounting Equations (2)-(5), Equation (1) can be represented as:

$$
\alpha\left(m_{x} \frac{\partial^{2} m_{y}}{\partial z^{2}}-m_{y} \frac{\partial^{2} m_{x}}{\partial z^{2}}\right)-h m_{y}-\left(\eta_{y}+\beta\right) m_{x} m_{y}=0
$$


with boundary conditions

$$
m_{x}=-1 \text { at } z=0 \text { and } \partial m_{x} / \partial z=0 \text { at } z=d
$$

In Equation (6), the material parameter $\beta$ represents the constant of single-axis anisotropy; $h=H / M$. Using the coupling between the magnetization components $m_{x}$ $=\cos (\chi)$ and $m_{y}=\sin (\chi)$, the Equation (6) can be rewritten in the following form:

$$
\frac{d^{2} \chi}{d z^{2}}+\frac{h}{\alpha} \sin \chi(1+b \cos \chi)=0
$$

with the boundary conditions for the angle $\chi$ in the case of transversal loading of a magnetic field (see Figure 1)

$$
\chi=\pi / 2 \text { at } z=0 \text { and } \partial \chi / \partial z=0 \text { at } z=d
$$

That leads to the equation of oscillations of the mathematical pendulum in the simplified case of $b=\left(\eta_{y}+\beta\right) / h$ $=0\left(\eta_{y} \rightarrow 0\right.$ and $\left.\beta \rightarrow 0\right)$ :

$$
\frac{d^{2} \chi}{d f^{2}}+q^{2} \sin \chi=0
$$

In Equation (10), the $f$ represents the normalized layer thickness, $f=z / d$, and $q^{2}=d^{2} h / \alpha$.

It is now necessary to separate variables such as $\chi$ and $f$. Setting intermediate variable $T=\mathrm{d} \chi / \mathrm{d} f$, it is possible to get the following equation $T \mathrm{~d} T / \mathrm{d} f=-q^{2} \sin (\chi)[1+b \cos (\chi)]$ resulting in $T^{2}=2 q^{2} \cos (\chi)[1+0.5 b \cos (\chi)]+$ const, and hence $\mathrm{d} \chi / \mathrm{d} f=\operatorname{sqrt}\left\{\right.$ const $\left.+2 q^{2} \cos (\chi)[1+0.5 b \cos (\chi)]\right\}$. Using the well-known trigonometric formula $\cos (\chi)=1-$ $2 \sin ^{2}(\chi / 2)$, it is possible to write an intermediate result:

$$
\mathrm{d} f=\frac{2 \mathrm{~d} \psi}{C_{1} \sqrt{1-k^{* 2}\left(1+b \cos ^{2} \psi\right) \sin ^{2} \psi}}
$$

with $C_{1}^{2}=(2+b) q^{2}+$ const, $\psi=\chi / 2$ and the parameter $k^{* 2} \equiv 1 / k^{2}$. The complicated case of Equation (11) is discussed below. In the simplified case of $b=0$, the constant $C_{1}{ }^{2} \rightarrow C^{2}=4 q^{2} k^{2}=2 q^{2}+$ const and

$$
k q \mathrm{~d} f=\frac{\mathrm{d} \psi}{\sqrt{1-k^{* 2} \sin ^{2} \psi}}
$$

In order to obtain the function $\psi(f)$, the right side of equality (12) can be written in the form of the elliptic integral of the first kind:

$$
k q f=F\left(\psi, k^{*}\right)+C^{*}
$$

where $C^{*}$ is a constant which should be determined from the boundary conditions. The elliptic integral of the first kind can be calculated with the descending Landen transformation [15]. Applying the boundary condition (9) at $z=0(f=0)$ and using the transformation formulas $F(\psi$, $\left.k^{*}\right)=k F\left(\psi^{*}, k\right)$ and $\sin (\psi)=k \sin \left(\psi^{*}\right)$ for $k^{* 2}=1 / k^{2}[14,15]$, the constant $C^{*}$ can be found as a function of $k$ : $C^{*}=$ $k F_{k}(k)$ with

$$
F_{k}(k)=F(\arcsin (\sqrt{2} / 2 k), k)
$$

Equation (13) can then be written as

$$
F\left(\arcsin \left(\frac{\sin \psi}{k}\right), k\right)=q f+F_{k}(k)
$$

and applying the function such as sine to both sides of Equation (15), one can get

$$
\sin \psi=k \operatorname{sn}(u, k)
$$

where $u=q f+F_{k}(k)$ and $\operatorname{sn}(u, k)$ is the elliptic sine representing one of the twelve Jacobian elliptic functions [15]. The function $\operatorname{sn}(u, k)$ can be also calculated with the descending Landen transformation [15]. Note that $F(\varphi, k)=u$ and $\varphi=\operatorname{am}(u)$ from $[14,15]$. Hence

$$
\chi=2 \arcsin [k \operatorname{sn}(u, k)]
$$

Applying the boundary condition (9) at $z=d(f=1)$ the parameter $q$ can be also obtained as a function of $k$ from the following equality:

$$
\frac{\mathrm{d} \chi}{\mathrm{d} f}=2 k q \mathrm{cn}(u, k)
$$

It is apparent that $\mathrm{d} \chi / \mathrm{d} f=0$ if the elliptic cosine $\operatorname{cn}(u, k)$ $=0$ that is satisfied for $u=\rho K(k)$ with $\rho=2 \tau-1$ and $\tau=$ $1,2,3, \ldots$, where $K(k)=F(\pi / 2, k)$ is called the complete elliptic integral of the first kind. Therefore

$$
q^{2}=d^{2} h / \alpha=\left(\rho K(k)-F_{k}(k)\right)^{2}
$$

and

$$
u=\left(\rho K(k)-F_{k}(k)\right) f+F_{k}(k)
$$

Note that the parameter $k^{2}$ should be confined in the following range:

$$
1 / 2 \leq k^{2} \leq 1
$$

The exact solutions for the magnetization components $m_{x}$ and $m_{y}$ can be written as functions of the Jacobian elliptic functions $\operatorname{sn}(u, k)$ and $\operatorname{dn}(u, k)$ in the following complicated form:

$$
\begin{gathered}
m_{x}=-2 k[\operatorname{dn}(u, k) \operatorname{sn}(u, k)] \\
m_{y}=-1+2 k^{2} \operatorname{sn}^{2}(u, k)
\end{gathered}
$$

where $\operatorname{dn}(u, k)$ is called the elliptic delta-amplitude.

Using the effective magnetic field $H_{a}$ of anisotropy [13] defined as $h_{a}=\alpha(\pi / 2 d)^{2}$, Equation (19) can be written as

$$
h / h_{a}=(2 / \pi)^{2}\left(\rho K(k)-F_{k}(k)\right)^{2}
$$

The average values, $\left\langle m_{x}\right\rangle$ and $\left\langle m_{y}\right\rangle$, of the magnetization components (22) and (23) can then be written in the following form:

$$
\begin{aligned}
\left\langle m_{x}\right\rangle & =\frac{1}{d} \int_{0}^{d} m_{x}(z) \mathrm{d} z=-2 k \frac{\operatorname{cn}(u, k)-\operatorname{cn}\left(F_{k}(k), k\right)}{\rho K(k)-F_{k}(k)} \\
\left\langle m_{y}\right\rangle=\frac{1}{d} \int_{0}^{d} m_{y}(z) \mathrm{d} z=-f+ & \\
& \frac{E(\operatorname{am} u, k)-E(\arcsin (\sqrt{2} / 2 k), k)}{\rho K(k)-F_{k}(k)}
\end{aligned}
$$


where $E(\operatorname{am} u, k)$ represents the elliptic integral of the second kind.

The exact solutions (25) and (26) were first introduced in [19] describing the dynamic instability in the nonlinear problem of a cantilever. Figure 2 shows the dependence of the values of $\left\langle m_{x}\right\rangle$ and $\left\langle m_{y}\right\rangle$ on the normalized values of $h / h_{a}$ for the static mode and several dynamic modes. The exact values of $h / h_{a}$ calculated with formula (24) are listed in Table 1. It is thought that these tabulated values of $h / h_{a}$ can be useful for researchers to check accuracy of different numerical methods.

The behaviors of the magnetization components $m_{x}$ and $m_{y}$ as functions of the $f=z / d$ are shown in Figure 3 (static mode) and Figures 4, 5, 6, and 7 (several corresponding dynamic modes) for several values of the pa-rameter $k^{2}$. It is clearly seen in all the figures that the satisfaction of the boundary condition of $m_{x}(f=0)=-1$ occurs. It is also seen in Figures 5 and $\mathbf{6}$ that the minimum values of the component $m_{y}=-1$ are significantly closer to each other for the different values of $k^{2}$ and the larger values of the $f$. This can mean that the magnetization vector $\mathbf{M}$ can be aligned along the $y$-axis just below the layer surface for the large values of $\rho$ and $f \rightarrow 1$, and any values of $k^{2}$ in the range: $1 / 2 \leq k^{2} \leq 1$. Note that Figure 7 shows the limit case of $k^{2}=1$ for the fourth dynamic mode with $\rho=9$. It is noted for comparison that in [17] homogeneous magnetization distributions were shown in the case of the axial loading of magnetic field. The following section theoretically investigates the magnetization components $m_{x}$ and $m_{y}$ written in Formulas

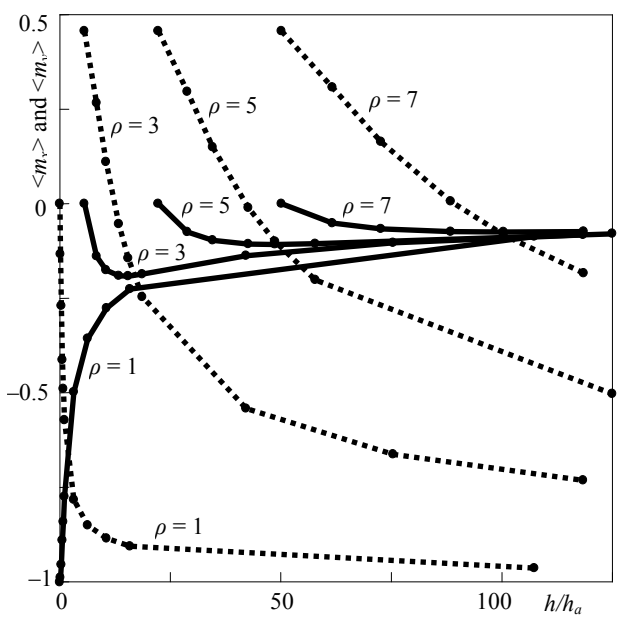

Figure 2. The dependence of the average magnetization components $\left\langle m_{x}\right\rangle$ and $\left\langle m_{y}\right\rangle$ on the values of $h / h_{a}$ for the static mode $(\rho=1)$ and three dynamic modes $(\rho=3,5,7)$.

Table 1. The values of $h / h_{a}$ with a high accuracy calculated with formula (24) for the static mode $(\rho=1)$ and four dynamic $\operatorname{modes}(\rho=3,5,7,9)$.

\begin{tabular}{|c|c|c|c|c|c|}
\hline \multirow{2}{*}{$k^{2}$} & \multicolumn{5}{|c|}{$h / h_{a}$} \\
\hline & $\tau=1, \rho=1$ & $\tau=2, \rho=3$ & $\tau=3, \rho=5$ & $\tau=4, \rho=7$ & $\tau=5, \rho=9$ \\
\hline 0.5 & 0.0 & 5.57281572 & 22.29126287 & 50.15534147 & 89.16505150 \\
\hline 0.6 & 0.16567298 & 8.34803259 & 28.85368867 & 61.68264124 & 106.83489028 \\
\hline 0.7 & 0.35546612 & 10.48881628 & 34.58708571 & 72.65027440 & 124.67838235 \\
\hline 0.8 & 0.61298524 & 13.37289437 & 42.65213110 & 88.45069543 & 150.76858736 \\
\hline 0.85 & 0.80045211 & 15.49579728 & 48.69611846 & 100.40141566 & 170.61168887 \\
\hline 0.9 & 1.08028952 & 18.67880751 & 57.82731652 & 118.52581656 & 200.77430761 \\
\hline 0.99 & 3.19667416 & 42.16367438 & 125.41284831 & 252.94419597 & 424.75771734 \\
\hline 0.999 & 6.35289569 & 75.41909073 & 220.47314024 & 441.51504424 & 738.54480272 \\
\hline 0.9999 & 10.58349145 & 118.41697367 & 342.64545246 & 683.26892783 & 1140.28739976 \\
\hline 0.99999 & 15.88920579 & 171.10175649 & 491.73272506 & 977.78211149 & 1629.24991578 \\
\hline
\end{tabular}




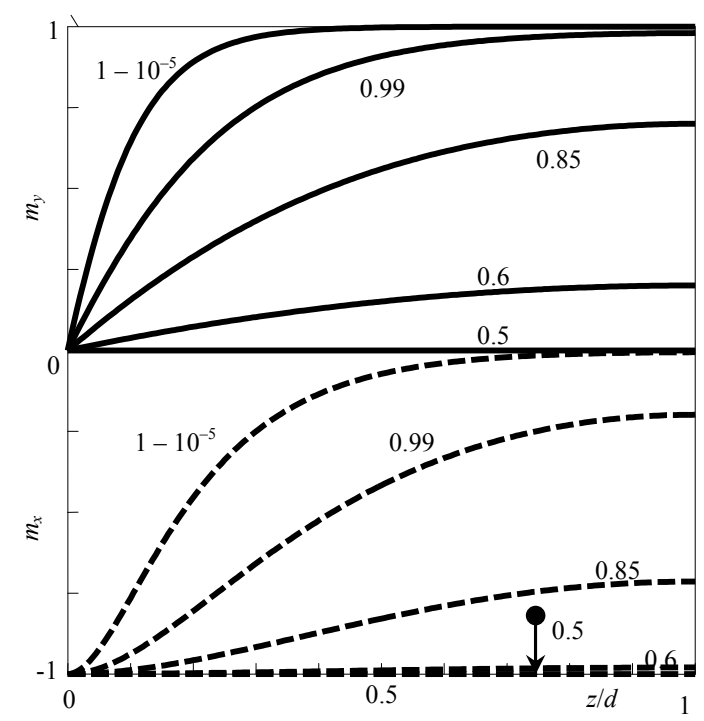

Figure 3. The magnetization distribution for the configuration of a magnetically-soft layer on a magnetically-hard layer versus the value of $z / d$; the static mode with $\rho=1$. Several values of the parameter $k^{2}$ are shown: $k^{2}=0.5,0.6$, $0.85,0.99,1-10^{-5}$.

(22) and (23) concerning study of their derivatives with respect to $f=z / d$.

\section{Derivatives of the Magnetization Components}

The first derivatives of the magnetization components $m_{x}$ and $m_{y}$ with respect to $f=z / d$ read:

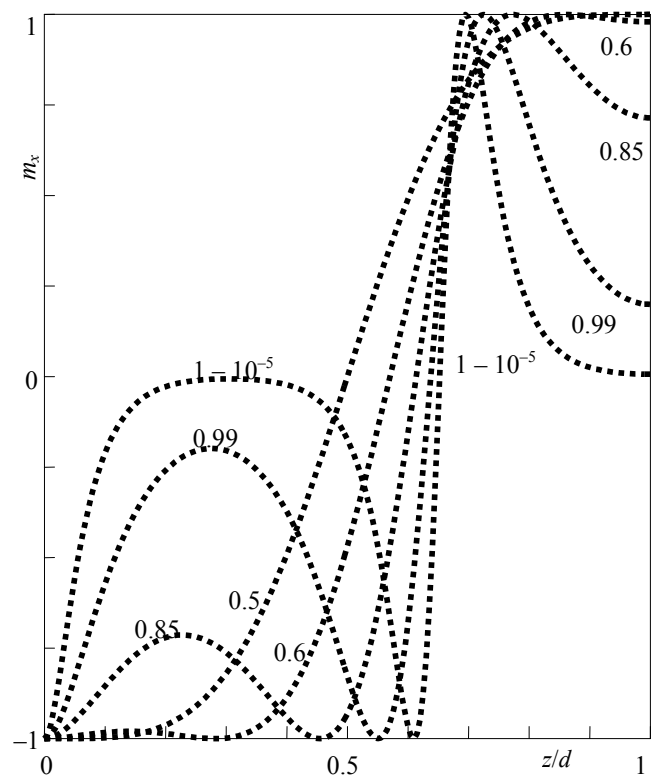

$$
\begin{aligned}
& \frac{\mathrm{d} m_{x}}{\mathrm{~d} f}=\frac{\mathrm{d} m_{x}}{\mathrm{~d} u} \frac{\mathrm{d} u}{\mathrm{~d} f} \\
& \frac{\mathrm{d} m_{y}}{\mathrm{~d} f}=\frac{\mathrm{d} m_{y}}{\mathrm{~d} u} \frac{\mathrm{d} u}{\mathrm{~d} f}
\end{aligned}
$$

where

$$
\mathrm{d} u / \mathrm{d} f=\rho K(k)-F_{k}(k)
$$

Note that the derivative $\mathrm{d} u / \mathrm{d} f$ in Equation (29) is equal to a constant for each $\rho$ and $k$. In Equations (27) and (28), the first derivatives of the $m_{x}$ and $m_{y}$ with respect to the function $u$ can be written as follows:

$$
\begin{aligned}
& \frac{\mathrm{d} m_{x}}{\mathrm{~d} u}=-2 k\left[\operatorname{dn}(u, k) \frac{\mathrm{dsn}(u, k)}{\mathrm{d} u}+\operatorname{sn}(u, k) \frac{\operatorname{ddn}(u, k)}{\mathrm{d} u}\right]= \\
& -(2 k) \operatorname{cn}(u, k)\left[2 \mathrm{dn}^{2}(u, k)-1\right]
\end{aligned}
$$$$
\frac{\mathrm{d} m_{y}}{\mathrm{~d} u}=4 k^{2} \operatorname{sn}(u, k) \frac{\mathrm{dsn}(u, k)}{\mathrm{d} u}=4 k^{2} \operatorname{sn}(u, k) \operatorname{cn}(u, k) \operatorname{dn}(u, k)
$$

where

$$
\begin{gathered}
\frac{\operatorname{dsn}(u, k)}{\mathrm{d} u}=\operatorname{cn}(u, k) \operatorname{dn}(u, k) \\
\frac{\operatorname{ddn}(u, k)}{\mathrm{d} u}=-k^{2} \operatorname{cn}(u, k) \operatorname{sn}(u, k)
\end{gathered}
$$

Using Equations (27) and (28), the second derivatives of the $m_{x}$ and $m_{y}$ with respect to the $f$ can be written as follows:

$$
\frac{\mathrm{d}^{2} m_{x}}{\mathrm{~d} f^{2}}=\frac{\mathrm{d} u}{\mathrm{~d} f} \frac{\mathrm{d}}{\mathrm{d} f}\left(\frac{\mathrm{d} m_{x}}{\mathrm{~d} u}\right)+\frac{\mathrm{d} m_{x}}{\mathrm{~d} u} \frac{\mathrm{d}^{2} u}{\mathrm{~d} f^{2}}=\frac{\mathrm{d}^{2} m_{x}}{\mathrm{~d} u^{2}}\left(\frac{\mathrm{d} u}{\mathrm{~d} f}\right)^{2}
$$

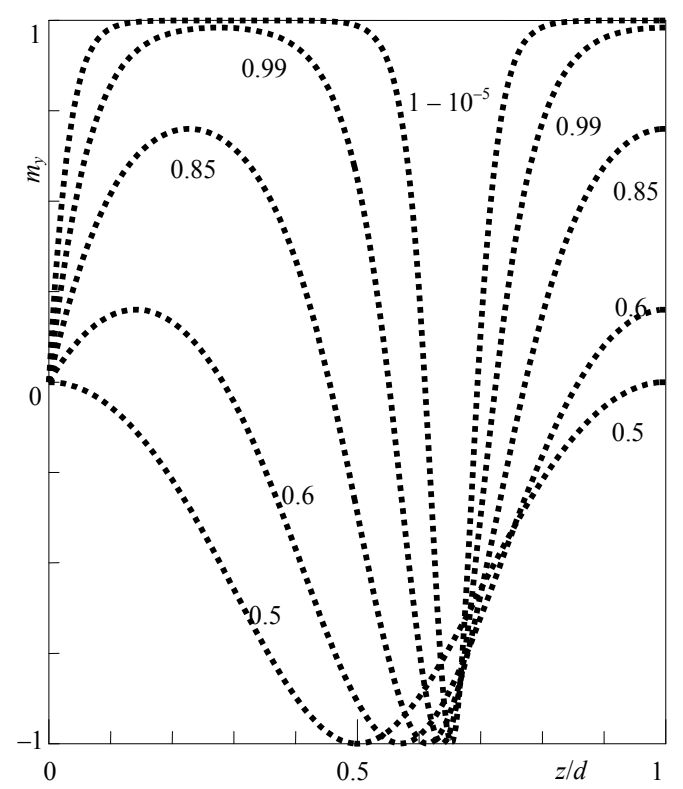

Figure 4. The magnetization components $m_{x}$ and $m_{y}$ versus the value of $z / d$; the first dynamic mode with $\rho=3$. The values of parameter $\boldsymbol{k}^{2}$ are the same to those for the static mode shown in Figure 3. 

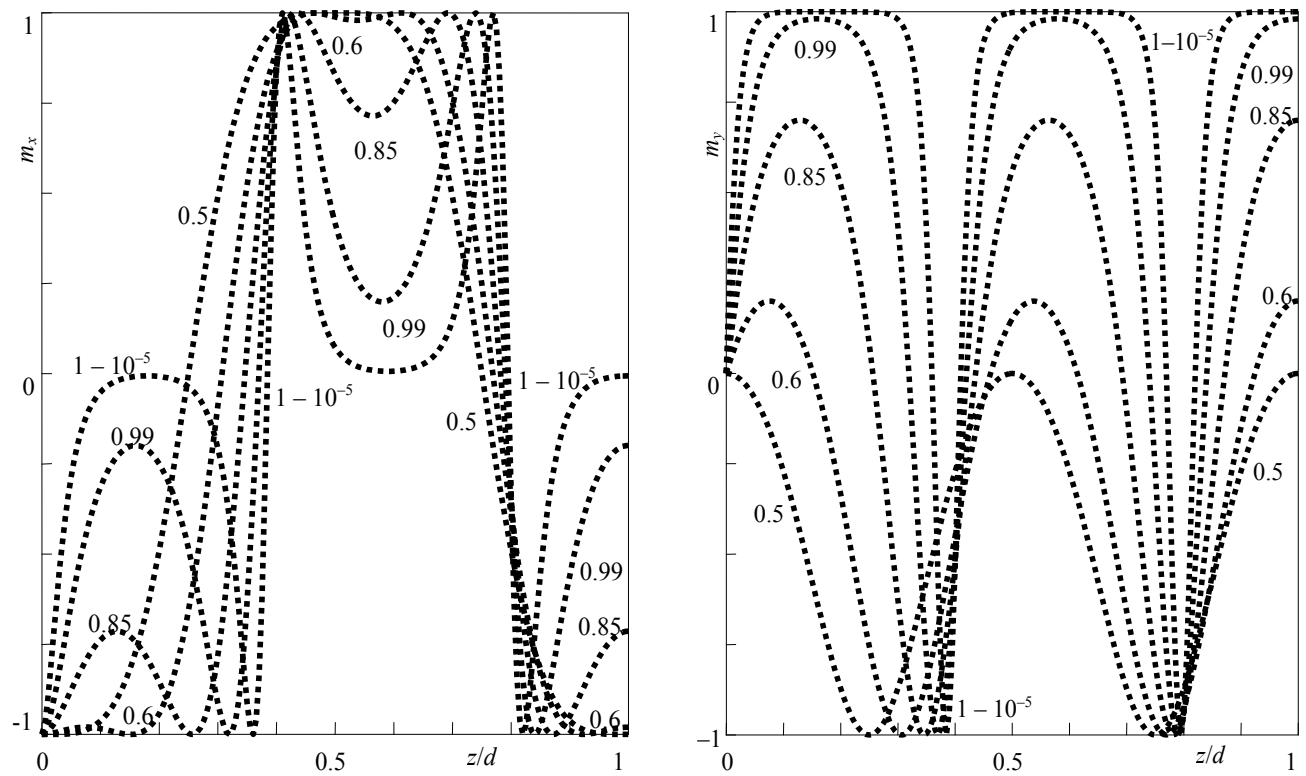

Figure 5. The magnetization components $m_{x}$ and $m_{y}$ versus the value of $z / d$; the second dynamic mode with $\rho=5$. The values of parameter $\boldsymbol{k}^{2}$ are the same to those for the static mode shown in Figure 3.
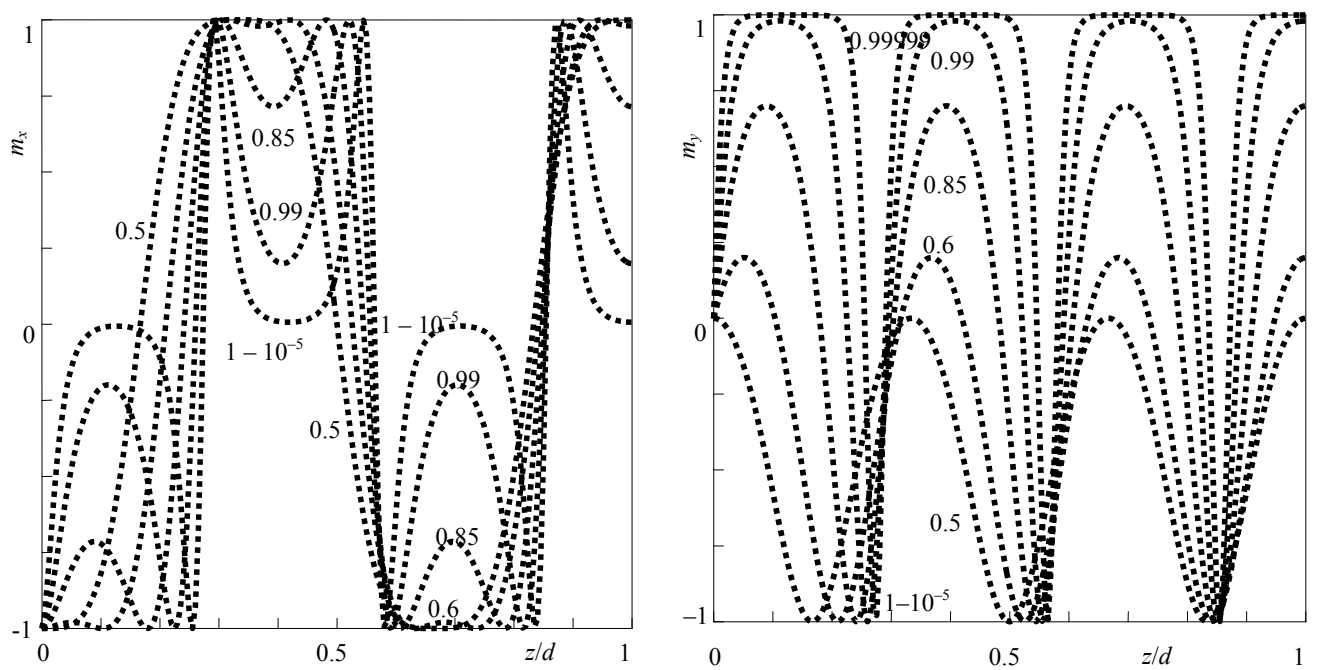

Figure 6. The magnetization components $m_{x}$ and $m_{y}$ versus the value of $z / d$; the third dynamic mode with $\rho=7$. The values of parameter $k^{2}$ are the same to those for the static mode shown in Figure 3.

$$
\frac{\mathrm{d}^{2} m_{y}}{\mathrm{~d} f^{2}}=\frac{\mathrm{d} u}{\mathrm{~d} f} \frac{\mathrm{d}}{\mathrm{d} f}\left(\frac{\mathrm{d} m_{y}}{\mathrm{~d} u}\right)+\frac{\mathrm{d} m_{y}}{\mathrm{~d} u} \frac{\mathrm{d}^{2} u}{\mathrm{~d} f^{2}}=\frac{\mathrm{d}^{2} m_{y}}{\mathrm{~d} u^{2}}\left(\frac{\mathrm{d} u}{\mathrm{~d} f}\right)^{2}
$$

It is stressed that the second derivative of the function $u$ with respect to $f$ in Equations (34) and (35) equals zero due to the linear dependence $u(f)$ because $K(k)$ and $F_{k}(k)$ are constants. In Equations (34) and (35), the second derivatives of the $m_{x}$ and $m_{y}$ with respect to the function $u$ can be written in the following form:

$$
\frac{\mathrm{d}^{2} m_{x}}{\mathrm{~d} u^{2}}=-2 k\left[\operatorname{dn}(u, k) \frac{\mathrm{d}^{2} \operatorname{sn}(u, k)}{\mathrm{d} u^{2}}+\right.
$$$$
\begin{gathered}
\left.\operatorname{sn}(u, k) \frac{\mathrm{d}^{2} \mathrm{dn}(u, k)}{\mathrm{d} u^{2}}+2 \frac{\mathrm{dsn}(u, k)}{\mathrm{d} u} \frac{\mathrm{ddn}(u, k)}{\mathrm{d} u}\right] \\
\frac{\mathrm{d}^{2} m_{y}}{\mathrm{~d} u^{2}}=4 k^{2}\left[\left(\frac{\mathrm{dsn}(u, k)}{\mathrm{d} u}\right)^{2}+\operatorname{sn}(u, k) \frac{\mathrm{d}^{2} \operatorname{sn}(u, k)}{\mathrm{d} u^{2}}\right]
\end{gathered}
$$

where

$$
\begin{aligned}
& \frac{\mathrm{d}^{2} \operatorname{sn}(u, k)}{\mathrm{d} u^{2}}=-\operatorname{sn}(u, k)\left[\operatorname{dn}^{2}(u, k)+k^{2} \operatorname{cn}^{2}(u, k)\right] \\
& \frac{\mathrm{d}^{2} \mathrm{dn}(u, k)}{\mathrm{d} u^{2}}=-k^{2} \operatorname{dn}(u, k)\left[\operatorname{cn}^{2}(u, k)-\operatorname{sn}^{2}(u, k)\right]
\end{aligned}
$$




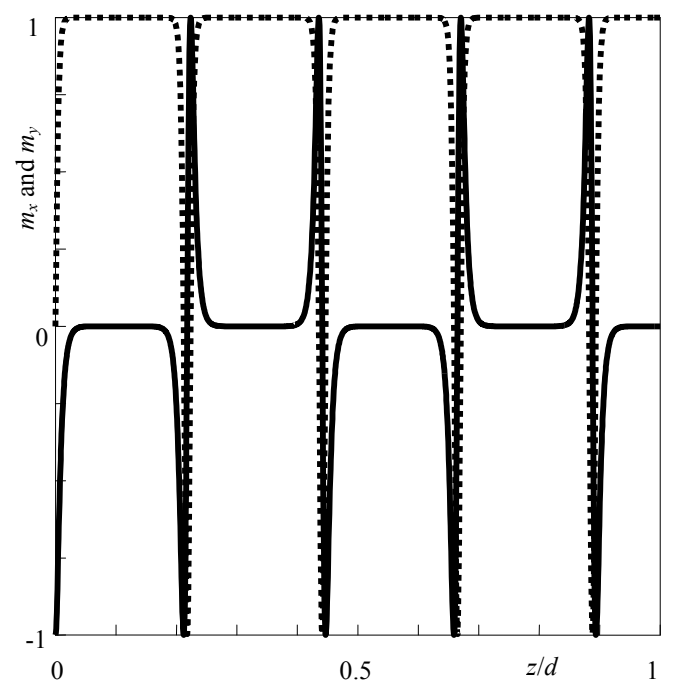

Figure 7. The magnetization components $m_{x}$ (solid line) and $m_{y}$ (doted line) versus the value of $z / d$; the fourth dynamic mode with $\rho=9$. The value of parameter $k^{2}$ equals to 1 .

In the same manner, it is possible here to write all derivatives of the $m_{x}$ and $m_{y}$ with respect to the $f$ :

$$
\begin{aligned}
\frac{\mathrm{d}^{n} m_{x}}{\mathrm{~d} f^{n}} & =\frac{\mathrm{d}^{n} m_{x}}{\mathrm{~d} u^{n}}\left(\frac{\mathrm{d} u}{\mathrm{~d} f}\right)^{n} \\
\frac{\mathrm{d}^{n} m_{y}}{\mathrm{~d} f^{n}} & =\frac{\mathrm{d}^{n} m_{y}}{\mathrm{~d} u^{n}}\left(\frac{\mathrm{d} u}{\mathrm{~d} f}\right)^{n}
\end{aligned}
$$

where the index $n$ is an integer and $n>0$.

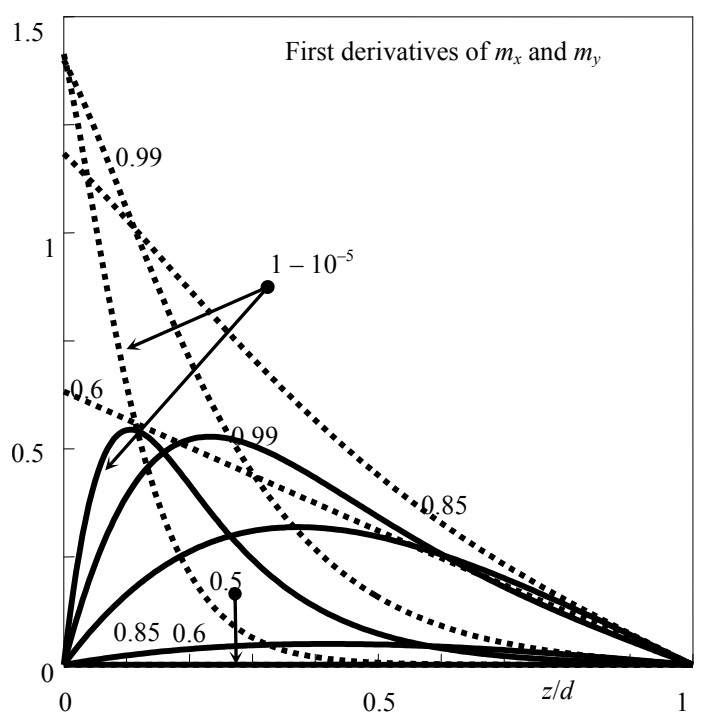

The first and second derivatives of the $m_{x}$ and $m_{y}$ are shown in Figure 8 for the static mode with $\rho=1$. It is clearly seen in Figure 8 that the first derivatives of the $m_{x}$ commence with zero values at $f=0$ and they together with the first derivatives of the $m_{y}$ become equal to zero at $f=1$. The second derivatives of the $m_{x}$ and $m_{y}$ for the static mode shown in Figure 8 were calculated with formulae (36)-(39). For the first dynamic mode, the first and second derivatives of the $m_{x}$ and $m_{y}$ are shown in Figures 9 and 10, respectively, as the functions of the values of $z / d$. It is seen in Figure 9 that the first derivatives of the $m_{x}$ commence with zero at $f=0$ and the derivatives of the $m_{x}$ and $m_{y}$ become equal to zero at $f=1$ that is similar to the case of the static mode.

\section{Non-Zero Value of the Parameter $B$ and Discussions}

In the case of non-zero parameter $b$, the magnetization components $m_{x}$ and $m_{y}$ were recently written as functions of the Jacobian elliptic functions $\operatorname{sn}(u, k)$ and $\operatorname{dn}(u, k)$ in the following complicated form [13]:

$$
\begin{gathered}
m_{x}=-2 \sqrt{k^{2}-\zeta^{2}} \operatorname{dn}(u, k) \operatorname{sn}(u, k) /\left(1-\zeta^{2} \operatorname{sn}^{2}(u, k)\right) \\
m_{y}=1-2 \operatorname{dn}^{2}(u, k) /\left(1-\zeta^{2} \operatorname{sn}^{2}(u, k)\right)
\end{gathered}
$$

According to [13], the parameter $\zeta$ represents a function of both parameters $k$ and $b$, which are independent of each other. The parameter $\zeta$ can have any value from 0 to 1 , and the parameter $b$ can be written as the following function of the $k$ and $\zeta[13]: b(k, \zeta)=\zeta^{2} /\left(k_{0}^{2}-2 k_{0}^{2} \zeta^{2}+\zeta^{2}\right)$ with $k_{0}^{2}=\left(k^{2}-\zeta^{2}\right) /\left(1-\zeta^{2}\right)$.

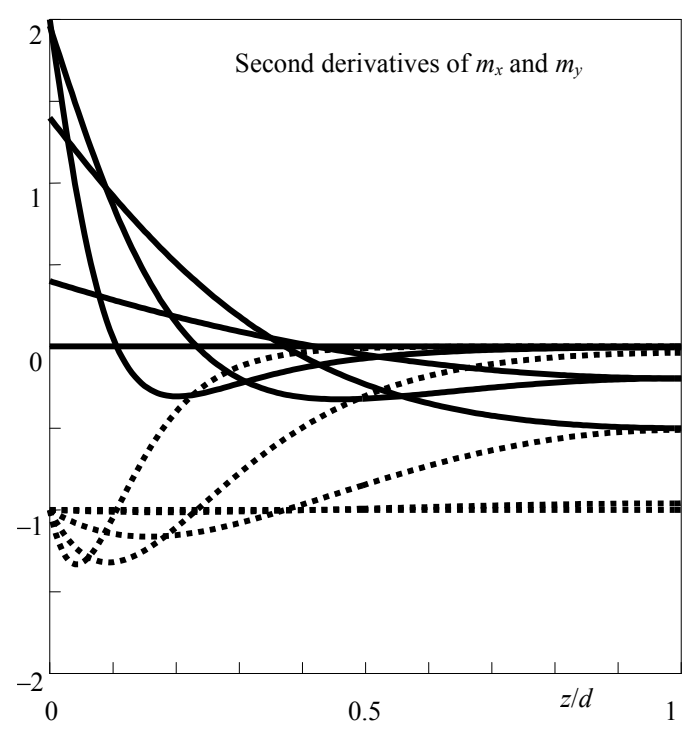

Figure 8. The first and second derivatives of $m_{x}$ (solid line) and $m_{y}$ (doted line) with respect to the $u$ versus the value of $z / d$; the static mode with $\rho=1$. The values of parameter $k^{2}$ are the same to those for the static mode shown in Figure 3. The factors $\mathrm{d} u / \mathrm{d} f=\rho K(k)-F_{k}(k)$ for the first derivatives of $m_{x}$ and $m_{y}$ are as follows: $0,0.64,1.41,2.81,6.26$ for $k^{2}=0.5,0.6,0.85$, $0.99,1-10^{-5}$, respectively. The factors $(\mathrm{d} u / \mathrm{d} f)^{2}$ for the second derivatives of $m_{x}$ and $m_{y}$ are as follows: $0,0.41,1.98,7.89,39.2$ for the corresponding $k^{2}$. 

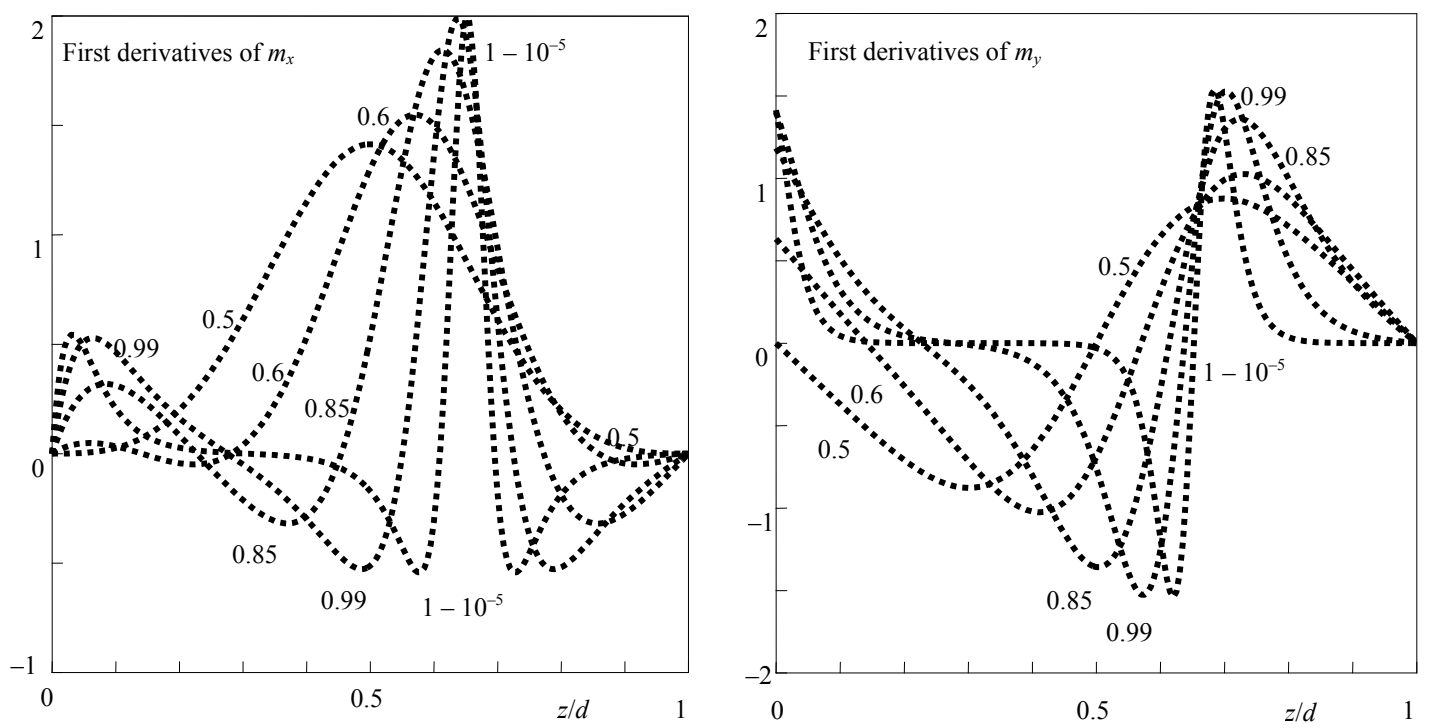

Figure 9. The first derivatives of $m_{x}$ and $m_{y}$ with respect to the $u$ versus the value of $z / d$; the first dynamic mode with $\rho=3$. The values of parameter $k^{2}$ are the same to those for the static mode shown in Figure 3. The factors $\mathrm{d} u / \mathrm{d} f=\rho K(k)-F_{k}(k)$ for the first derivatives of $m_{x}$ and $m_{y}$ are as follows: 3.71, 4.54, 6.18, 10.2, 20.5 for $k^{2}=0.5,0.6,0.85,0.99,1-10^{-5}$, respectively.
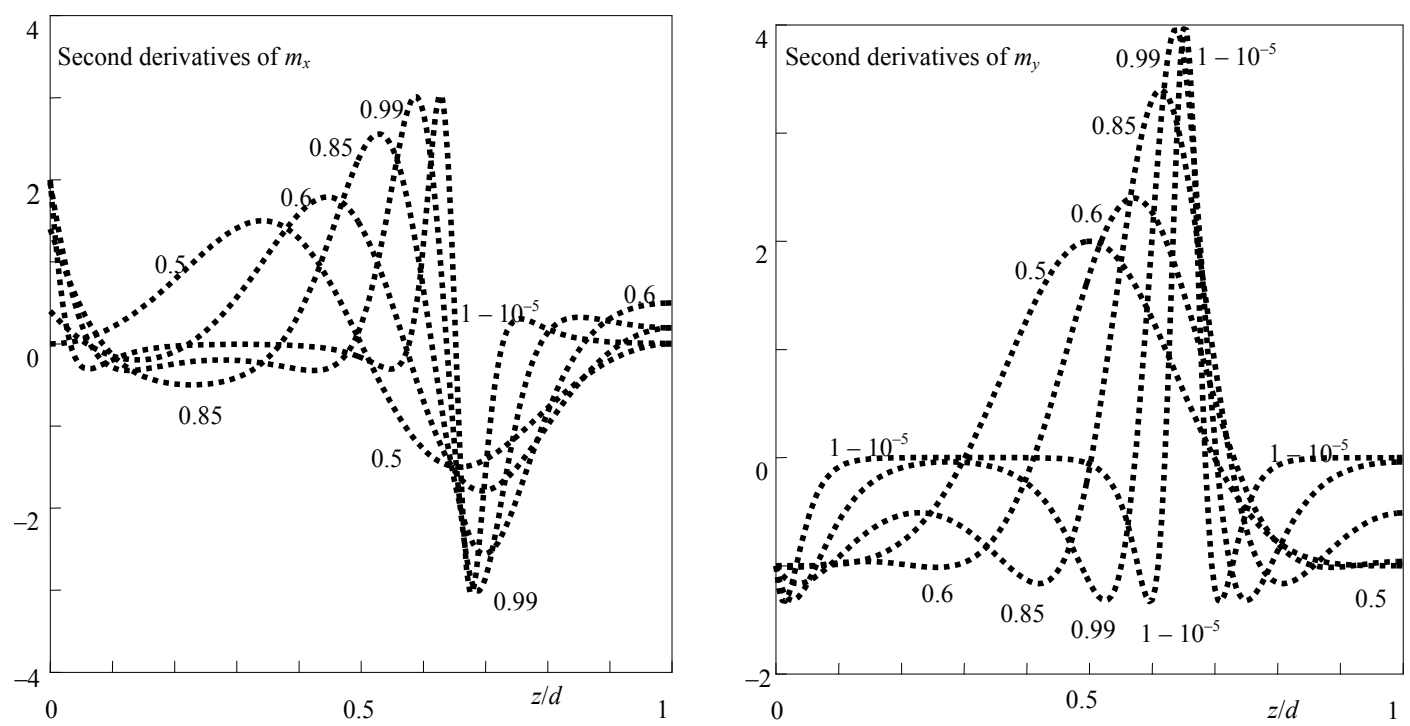

Figure 10. The second derivatives of $m_{x}$ and $m_{y}$ with respect to the $u$ versus the value of $z / d$; the first dynamic mode with $\rho=3$. The values of parameter $k^{2}$ are the same to those for the static mode shown in Figure 3. The factors $(\mathrm{d} u / \mathrm{d} f)^{2}$ for the second derivatives of $m_{x}$ and $m_{y}$ are as follows: 13.8, 20.6, 38.2, 104, 422 for $k^{2}=0.5,0.6,0.85,0.99,1-10^{-5}$, respectively.

Zakharov and Khlebopros [13] represented solutions (42) and (43) as the exact solutions for the magnetization components $m_{x}$ and $m_{y}$ in the case of the axial loading of the magnetic field $\mathbf{H}$. Indeed, in the case of the axial loading, they satisfy the boundary condition $m_{y}(b \neq 0, f=$ $0)=m_{y}(b=0, f=0)=-1$ and the relationship between the components: $m_{x}{ }^{2}+m_{y}{ }^{2}=1$. It is thought that the following must be fulfilled: $m_{y}(b \neq 0, f=1)=m_{y}(b=0, f=$ 1) because $\cos (\psi=\pi / 2)=0$ at $f=1$ in Equation (11). However, that is also not fulfilled using solutions (42) and (43). Note that in the case of the axial loading of the
$\mathbf{H}$, the magnetization vector $\mathbf{M}$ should be directed towards negative values of the $y$-axis that is anti-parallel to the vector $\mathbf{H}$. It was also found that in the case of transversal loading of the magnetic field, solutions (42) and (43) can satisfy only the relationship $m_{x}{ }^{2}+m_{y}{ }^{2}=1$, because it should be true for any $u$ and $k$. In this case of transversal loading according to the boundary condition at $f=0$, the magnetization component $m_{x}$ should equal to -1 . However, that does not occur for any non-zero parameter $\zeta$, using Equations (42) and (43). It is thought that any solution of the problem in both cases of the 
transversal and axial loading for $b \neq 0$ should satisfy the boundary conditions similar to what occurs in the case of $b=0$, using Equations (22) and (33) in both cases of the transversal and axial loading of the magnetic field $\mathbf{H}$. Therefore, one method is offered in this paper below to numerically obtain solutions for the case of $b \neq 0$, which entirely satisfy the boundary conditions at $f=0$ and $f=1$ for the cases of the transversal and axial loading such as in the problem of $b=0$.

Equation (11) can be written as follows:

$$
C_{1} \mathrm{~d} f=\frac{2 \mathrm{~d} \psi}{\sqrt{1-k^{* 2} A^{2} \sin ^{2} \psi}}
$$

with $C_{1}^{2}=(2+b) q^{2}+$ const, $\psi=\chi / 2$ and the function $A^{2}$ $=1+b \cos ^{2}(\psi)$. Here, it is assumed that in Equation (44), the function $A$ results in the parameter $k^{*}$ that is convenient in order to cope with an integral in the form of the elliptic integral of the first kind. Hence, Equation (44) can be written as:

$$
k_{b} q A \mathrm{~d} f=\frac{\mathrm{d} \psi}{\sqrt{1-k_{b}^{* 2} \sin ^{2} \psi}}
$$

with the parameter $k_{b}{ }^{* 2}=k^{* 2} A^{2}$ and $k_{b}{ }^{* 2} \equiv 1 / k_{b}{ }^{2}$, hence $k_{b}{ }^{2}$ $=k^{2} / A^{2}$. Note that in this case in Equations (44) and (45), the constant $C_{1}^{2}=4 q^{2} k^{2}=4 q^{2} k_{b}^{2} A^{2}$ represents a function of the parameter $b$ and angle $\psi$, but it should also remain a constant.

It is thought that the following mathematical transformations can be written in the same manner as Formulas (13) to (23): the right side of equality (45) can be also written in the form of the elliptic integral of the first kind:

$$
k_{b} q A f=F\left(\psi, k_{b}^{*}\right)+C^{*}
$$

where $C^{*}$ is a constant which is also determined from the boundary conditions. Applying the boundary condition (9) at $f=0$ and using the transformations $F\left(\psi, k_{b}{ }^{*}\right)=$ $k_{b} F\left(\psi^{*}, k_{b}\right)$ and $\sin (\psi)=k_{b} \sin \left(\psi^{*}\right)$ for $k_{b}{ }^{* 2}=1 / k_{b}{ }^{2}$, the constant $C^{*}$ is analogically found as follows: $C^{*}=$ $k_{b} F_{k b}\left(k_{b}\right)$ with

$$
F_{k b}\left(k_{b}\right)=F\left(\arcsin \left(\sqrt{2} / 2 k_{b}\right), k_{b}\right)
$$

giving

$$
F\left(\arcsin \left(\frac{\sin \psi}{k_{b}}\right), k_{b}\right)=q A f+F_{k b}\left(k_{b}\right)
$$

Note that in Equation (48), the parameter $k_{b}$ is absent, and hence $q A$ represents a function, but not a constant. Indeed, it is also possible to apply a harmonic function such as sine to both sides of Equation (48) that results in the following:

$$
\sin \psi=k_{b} \operatorname{sn}\left(u_{b}, k_{b}\right)
$$

with $u_{b}=q A f+F_{k b}\left(k_{b}\right)$. It is also noted that $F\left(\varphi, k_{b}\right)=u_{b}$ and $\varphi=\operatorname{am}\left(u_{b}\right)$. Hence

$$
\chi=2 \arcsin \left[k_{b} \operatorname{sn}\left(u_{b}, k_{b}\right)\right]
$$

Utilizing boundary condition (9) at $f=1$, the parameter $q A$ is also obtained as a function of $k_{b}$.

It is apparent that $\mathrm{d} \chi / \mathrm{d} f=0$ if the elliptic cosine $\operatorname{cn}\left(u_{b}\right.$, $\left.k_{b}\right)=0$ that is satisfied in the case of $b \neq 0$ already for $u_{b}$ $=\rho K\left(k_{b}\right)$ with $\rho=2 \tau-1$ and $\tau=1,2,3, \ldots$. Therefore, it is possible to write the following result: $q^{2} A^{2}=$

$$
\begin{aligned}
& \left(\rho K\left(k_{b}\right)-F_{k b}\left(k_{b}\right)\right)^{2} \text {. Hence } \\
& \qquad u_{b}=\left(\rho K\left(k_{b}\right)-F_{k b}\left(k_{b}\right)\right) f+F_{k b}\left(k_{b}\right)
\end{aligned}
$$

The solutions for the $m_{x}$ and $m_{y}$ can be also written in the form of the Jacobian elliptic functions, namely $\operatorname{sn}\left(u_{b}\right.$, $\left.k_{b}\right)$ and $\operatorname{dn}\left(u_{b}, k_{b}\right)$ :

$$
\begin{gathered}
m_{x}=-2 k_{b}\left[\operatorname{dn}\left(u_{b}, k_{b}\right) \operatorname{sn}\left(u_{b}, k_{b}\right)\right] \\
m_{y}=-1+2 k_{b}{ }^{2} \operatorname{sn}^{2}\left(u_{b}, k_{b}\right)
\end{gathered}
$$

Using the effective magnetic field $h_{a}$ of anisotropy, it is possible to write as follows:

$$
h / h_{a}=(2 / \pi)^{2}\left(\rho K\left(k_{b}\right)-F_{k b}\left(k_{b}\right)\right)^{2} / A^{2}
$$

Note that solutions (52) and (53) for the case of $b \neq 0$ look like the exact solutions in Equations (22) and (23) for the case of $b=0$. Therefore, they should satisfy the boundary conditions at both $f=0$ and $f=1$. It is obvious that solutions (52) and (53) are formed from the exact solutions in Equations (22) and (23) by the following substitutions: $k \rightarrow k_{b}$ and $u \rightarrow u_{b}$. Note that in the case of $b \neq 0$, the parameter $k_{b}$ depends on both the parameter $b$ and the angle $\psi=\chi / 2$. Therefore, the angle $\psi$ depends on the $k_{b}(\psi)$ in Equation (49), so that as soon as the angle $\psi$ is changed, the $k_{b}(\psi)$ is also correspondingly changed. Indeed, it is necessary to apply the following recursive procedure using Equation (50): $\chi_{N+1}=2 \arcsin \left[k_{b}\left(\chi_{N}\right)\right.$ $\left.\operatorname{sn}\left(u_{b}\left(\chi_{N}\right), k_{b}\left(\chi_{N}\right)\right)\right] \quad(N=0,1,2, \ldots)$. The right angle $\chi$ is found when $\chi_{N+1}=\chi_{N}$. Fortunately, this numerical problem can be resolved. It is thought that for the numerical procedure to compute magnetization components (52) and (53), the exactly determined angle $\chi$ in the case of $b=0$ can be used as an initial guess $\chi_{0}$ to numerically find the right angle $\chi_{N}$ in Equation (50) for the case of $b \neq 0$. It was set in the numerical procedure to interrupt the calculation process when $\operatorname{abs}\left(\chi_{N+1}-\chi_{N}\right)<10^{-7}$. Note that such numerical calculations can be readily completed with a modern computer, for instance, a laptop with a 20-inch monitor and a four-core processor. It is also thought that this numerical method can be useful for finding solutions when the function $A$ represents more complicated function of the angle $\psi$, depending on several parameters $b_{i}$.

To compare the solutions for the cases of $b=0$ and $b \neq$ 0 , Figures 11 and 12 show the magnetization components $m_{x}$ and $m_{y}$ (transversal loading of a magnetic field) for the static mode $(\rho=1)$ and the first dynamic mode ( $\rho$ $=3$ ) respectively. It is possible to notice in the figures that in the case of the dynamic mode in Figure 12, the 


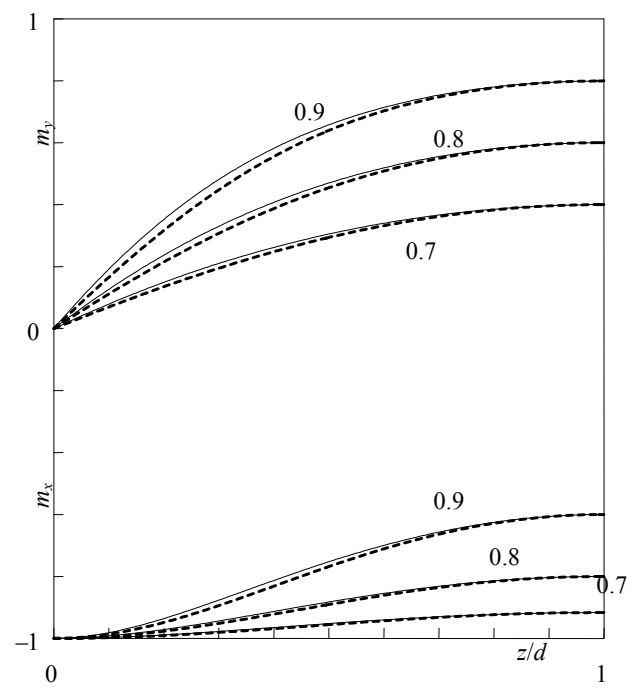

Figure 11. The magnetization components $m_{x}$ and $m_{y}$ versus the value of $z / d$ for the static mode $(\rho=1)$ for $k^{2}=0.7,0.8$, and 0.9 . Solid lines are for $b=0$ and the dashed lines are for $b \sim 0.158,0.139$, and 0.123 , respectively.

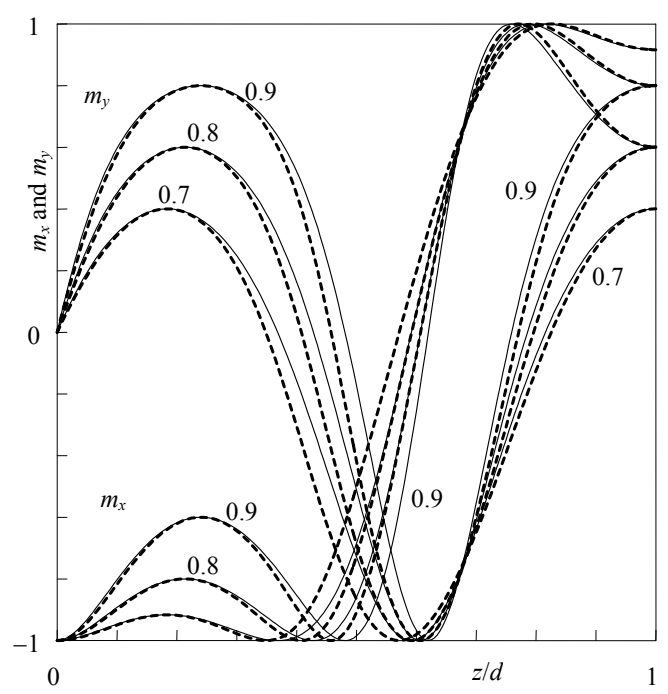

Figure 12. The magnetization components $m_{x}$ and $m_{y}$ versus the value of $z / d$ for the first dynamic mode $(\rho=3)$ for $k^{2}=$ $0.7,0.8$, and 0.9 . Solid lines are for $b=0$ and the dashed lines are for $b \sim 0.158,0.139$, and 0.123 , respectively.

difference between the cases of $b=0$ (solid line) and $b \neq$ 0 (dashed line) is more significant than that for the case of the static mode in Figure 11. The figures show the magnetization behaviors for relatively small values of $b$ $<0.2$. Also, it is clearly seen that for the dynamic mode, the values of the components $m_{x}$ and $m_{y}$ reach -1 at the smaller values of $z / d$ for the case of $b \neq 0$.

\section{Conclusions}

This paper demonstrated the magnetization distribution in a magnetically-soft layer (ferromagnetics) on a magnetically-hard substrate (anti-ferromagnetics) when the applied magnetic field is perpendicular to the initial magnetization. Solutions were written in the form of combination of the Jacobian elliptic functions and elliptic integrals. The average values of magnetization components, $\left\langle m_{x}\right\rangle$ and $\left\langle m_{y}\right\rangle$, were calculated in dependence on the applied magnetic field. The static mode and several dynamic modes of magnetization components $m_{x}$ and $m_{y}$ were also calculated in order to illuminate their distributions across the layer thickness. The first and second derivatives of the magnetization components were also calculated. Also, it was found that the inclusion of magnetic anisotropy $(b \neq 0)$ in calculations can complicate the finding of the magnetization components and show a significant difference. Note that the utilized solutions for the problem completely satisfy the boundary conditions applied to the magnetically-soft layer with inhomogeneous boundaries.

\section{References}

[1] L. Landau and E. Lifshits, "On the Theory of the Dispersion of Magnetic Permeability in Ferromagnetic Bodies," Physikalische Zeitschrift der Sowjetunion, Vol. 8, 1935, pp. 153-169.

[2] L. Landau and E. Lifshits, "On the Theory of the Dispersion of Magnetic Permeability in Ferromagnetic Bodies," Ukrainian Journal of Physics, Special Issue, Vol. 53, 2008, pp. 14-22.

[3] J. Li, "A Two-Dimensional Landau-Lifshitz Model in Studying Thin Film Micromagnetics," Abstract and Applied Analysis, Vol. 13, 2009.

[4] J.-N. Li, X.-F. Wang and Z.-A. Yao, "An Extension Landau-Lifshitz Model in Studying Soft Ferromagnetic Films," Acta Mathematicae Applicatae Sinica, English Series, Vol. 23, No. 3, 2007, pp. 421-432.

[5] L. G. Korzunin, B. N. Filippov and F. A. Kassan-Ogly, "Dynamics of Vortex-like Domain Walls in Triaxial Magnetic Films with the Goss Orientation of the Surface," Technical Physics, Vol. 52, No. 11, 2007, pp. 1453-1457.

[6] B. N. Filippov and L. G. Korzunin, "Non-linear Dynamics of Domain Walls with Vortex-like Inner Structure in Magnetically-single-axis Films with Plane Anisotropy," Journal of Experimental and Technical Physics, Moscow, Vol. 121, No. 2, 2002, pp. 372-387.

[7] P. L. Sulem, C. Sulem and C. Bardos, "On the Continuous Limit for a System of Classical Spins," Communications in Mathematical Physics, Vol. 107, No. 3, 1986, pp. 431-454.

[8] W. E and X.-P. Wang, "Numerical Methods for the Landau-Lifshitz Equation" SIAM Journal on Numerical Analysis, Vol. 38, No. 5, 2000, pp. 1647-1665.

[9] A. DeSimone, R. V. Kohn, S. Müller and F. Otto, "A Reduced Theory for Thin-Film Micromagnetics," Communications on Pure and Applied Mathematics, Vol. 55, 
No. 11, 2002, pp. 1408-1460.

[10] A. DeSimone, R. V. Kohn, S. Müller, F. Otto and R. Schäfer, "Two-Dimensional Modelling of Soft Ferromagnetic Films," Proceedings of the Royal Society of London, Series A, Vol. 457, No. 2016, 2001, pp. 29832991.

[11] A. Aharoni, E. H. Frei and S. Shtrikman, "Theoretical Approach to the Asymmetrical Magnetization Curve," Journal of Applied Physics, Vol. 30, No. 12, 1959, pp. 1956-1961.

[12] N. M. Salansky and M. S. Eruchimov, "The Peculiarities of Spin-Wave Resonance in Films with Ferro-Antiferromagnetic Interaction," Thin Solid Films, Vol. 6, No. 2, 1970, pp. 129-140.

[13] Y. V. Zakharov and E. A. Khlebopros, "Magnetization Curves and Frequencies of Magnetic Resonance in Films with Domain Structures on Anti-Ferromagnetic Substrate," Solid State Physics, Moscow, Vol. 22, No. 12, 1980, pp. 3651-3657.

[14] H. Bateman and A. Erd'elyi, "Higher Transcendental Functions," McGraw-Hill, New York, Vol. 3, 1955.

[15] M. Abramowitz and I. A. Stegun, Eds., "Handbook of Mathematical Functions with Formulas," Graphs and Mathematical Tables, National Bureau of Standards in Applied Mathematics, Washington, 1964, p. 1058.
[16] L. Collatz, "Eigenvalue Problems with Engineering Applications," Fizmatgiz, Moscow, 1968.

[17] Y. V. Zakharov, "Static and Dynamic Instability of a Ferromagnetic Layer under Magnetic Reversal," Doklady of Russian Academy of Science, Vol. 344, No. 3, 1995, pp. 328-332.

[18] M. A. Lavrent'ev and A. Y. Ishlinskii, "Dynamic Buckling Modes of Elastic Systems," Doklady Akademii Nauk USSR (Moscow), Vol. 64, No. 6, 1949, pp. 779-782.

[19] Y. V. Zakharov and A. A. Zakharenko, "Dynamic Instability in the Nonlinear Problem of a Cantilever," Computational Technologies, Vol. 4, No. 1, 1999, pp. 48-54.

[20] M. Johnson, "Bipolar Spin Switch," Science, Vol. 260 No. 5106, 1993, pp. 320-323.

[21] D. J. Monsma, J. C. Lodder, T. J. A. Popma and B. Dieny, "Perpendicular Hot Electron Spin-Valve Effect in a New Magnetic Field Sensor: The Spin-Valve Transistor," Physical Review Letters, Vol. 74, No. 26, 1995, pp. 52605263.

[22] M. Johnson, "Spin Injector in Metal Films: The Bipolar Spin Transistor," Materials Science and Engineering B, Vol. 31, 1995, pp. 199-205.

[23] V. Y. Shur and E. L. Rumyantsev, "Kinetics of Ferroelectric Domain Structure during Switching: Theory and Experiment," Ferroelectrics, Vol. 151, 1994, pp. 171-189. 